\title{
Contribuições do yoga frente a ansiedade e a qualidade de vida
}

\section{Contributions of yoga to anxiety and quality of life}

\author{
Shirlei Magda da Silva Souza ${ }^{1}$
}

Alexandre Silva de Mello²

\section{RESUMO}

Estamos vivendo um momento em que o número de pessoas com ansiedade está aumentando a cada dia em todo o mundo e segundo dados da OMS, Organização Mundial de Saúde, o Brasil é neste momento o país mais ansioso da América Latina. Estudos recentes têm sugerido que a pratica do Yoga tem efeitos positivos sobre quadros de estresse, ansiedade e depressão. Yoga é uma filosofia de vida fundamentada em princípios éticos, técnicos e filosóficos, com sua origem na índia. 0 Yoga trabalha o corpo, respiração e mente, abrange todos os aspectos da vida: físico, mental, emocional, psicológico e espiritual. Este estudo teve o objetivo de verificar o efeito da prática de Yoga frente a ansiedade e a qualidade de vida, para isso foram utilizados dois questionários: o inventário de ansiedade de Beck, que verificou o nível de ansiedade; o questionário Whoqol-Bref, que avaliou o grau de qualidade de vida das participantes. Ambos foram aplicados em dois momentos, antes de iniciar a prática de Yoga, e ao término da atividade. E um questionário socioeconômico para caracterização da amostra. A pesquisa foi realizada em uma Empresa de Advocacia, em Sapucaia do Sul. As voluntárias realizaram práticas de Yoga duas vezes por semana durante um mês e meio, no total de 12 aulas de Yoga. Este estudo se caracterizou por uma pesquisa de estudo longitudinal, comparativo de abordagem quantitativa. A amostra foi formada por conveniência e composta por 12 voluntárias mulheres funcionárias de uma empresa de direito, com queixa de ansiedade, sendo a maioria jovens com idade de 20 a 30 anos e uma pequena quantidade com idade de 40 a 52 anos. Nossos resultados foram positivos, após as práticas de Yoga, as participantes teve uma melhora significativa em relação a ansiedade e a qualidade de vida. 0 grau de ansiedade diminuiu consideravelmente demonstrando que o Yoga tem eficácia para uma vida saudável. Sendo assim, este estudo pode contribuir para o desenvolvimento de estratégias na diminuição da ansiedade e melhora na qualidade de vida das pessoas.

\section{PALAVRAS-CHAVE}

Yoga; Ansiedade; Qualidade de vida.

${ }^{1}$ Mestranda em Biociências e Reabilitação. Centro Universitário Metodista - IPA. Porto Alegre, RS. Brasil. E-mail: shirleimagss@gmail.com

${ }^{2}$ Centro Universitário Metodista - IPA. Porto Alegre, RS. Brasil. E-mail:melloas@gmail.com 


\section{ABSTRACT}

We are living in a time when the number of people with anxiety is increasing every day all over the world and according to data from WHO, World Health Organization, Brazil is currently the most anxious country in Latin America. Recent studies have suggested that the practice of Yoga has positive effects on stress, anxiety and depression. Yoga is a philosophy of life based on ethical, technical and philosophical principles, with its origin in India. Yoga works the body, breath and mind, covers all aspects of life: physical, mental, emotional, psychological and spiritual. This study aimed to verify the effect of Yoga practice in the face of anxiety and quality of life, two questionnaires were used: Beck's anxiety inventory, which checked the level of anxiety; the Whoqol-Brief questionnaire, which assessed the participants' level of quality of life. Both were applied in two moments, before starting the practice of Yoga, and at the end of the activity. The research was carried out at a Law Firm in Sapucaia do Sul. The volunteers performed Yoga practices twice a week for a month and a half, for a total of 12 Yoga classes. This study was characterized by a cross-sectional, comparative study with a quantitative approach.The sample was formed by convenience and consisted of 16 volunteer women employees of a law firm, complaining of anxiety, the majority being young people aged 20 to 30 years old and a small amount aged 40 to 52 years old. Our results were positive, after Yoga practices, the participants had a significant improvement in relation to anxiety and quality of life. The degree of anxiety has decreased considerably, demonstrating that Yoga is effective for a healthy life. There fore, this study can contribute to the development of strategies to reduce anxiety and improve people's quality of life.

\section{KEYWORDS}

Yoga; Anxiety; Qualityoflife. 


\section{INTRODUÇÃO}

Todos nós desde o nascimento, temos uma dose "terapêutica" de ansiedade, necessária a nossa sobrevivência. A ansiedade patológica surge em nossa vida como um sentimento de apreensão, uma sensação constante de que alguma coisa vai acontecer, inesperadamente. Quem sofre desta ansiedade patológica vive em contínuo estado de alerta e inquietude, deixa de viver o presente, pois preocupações subjetivas tomam o espaço do aqui agora. (SILVA, 2017).

Ossinaiseossintomasassociadosàansiedadedesencadeiam alterações psicofisiológicas que muitas vezes requerem tratamento por provocarem no indivíduo prejuízo no seu bem estar e também em seu desempenho sócio-ocupacional. A ansiedade clínica é muito mais grave que a tendência comum as preocupações do cotidiano. (GUIMARÃES,2007; LEAHY, 2010)

A sociedade atual já foi classificada como a sociedade do estresse e da ansiedade. Somos cobrados a todo o momento e em todas as circunstâncias de nossas vidas. Para algumas pessoas, a aquisição de um corpo perfeito passa ser uma obrigação e para outras a aquisição de status profissional é um pré-requisito para felicidade. Dependendo do nível de nossa cobrança, tais desejos podem produzir emoções desastrosas e dentre elas a ansiedade (DANUCALOV \& SIMÕES, 2009).

Essa cultura consumista e individualista está tão enraizada em nosso comportamento diário, que não percebemos o quanto vivemos a ditadura do ter. Por um lado os seres humanos nunca tiveram tanto acesso a bens materiais como hoje, e por outro, nunca tantas pessoas insatisfeitas, ansiosas e compulsivas por comida, compras, drogas, jogos, sexo, redes sociais, internet etc (SILVA, 2017).

O Brasil é considerado o país mais ansioso e estressado da América Latina. Segundo a Organização Mundial da Saúde (OMS), nos últimos dez anos o número de pessoas com depressão aumentou 18,4\%, isso corresponde a 322 milhões de indivíduos, ou 4,4\% da população da Terra. No Brasil, 5,8\% dos habitantes ? a maior taxa do continente latino-americano - sofrem com o problema (GRACIOLI,2018).

O Yoga vemcontra portudooque foi abordado nos parágrafos anteriores, o Yoga é um ato supremo de autocompreensão, é um mergulho profundo diário em nós mesmos, é a forma como você vivencia seu corpo e sua mente. Se sua mente está tensa, teu corpo está emdequilíbrio, se sua mente está calma, seu corpo e sua vida também refletirá. 0 Yoga gera um bem-estar, é uma prática importante que promove saúde para quem pratica (STILES,2014; BARROS et al,2014).

Segundo Borella et al. (2007), Yoga, termo sânscrito associado ao conceito e união,é um movimento cultural que se desenvolveu ao longo de milênios na região que hoje é a índia, é uma filosofia que visa a identificação do ser humano com sua própria natureza e sua integração com o universo.

SARASWATI conceitua o Yoga como a ciência do reto-viver e, assim, tem a intenção de ser incorporada na vida diária. Funciona em todos os aspectos de uma pessoa: psicológico, vital, mental, emocional, físico e espiritual (SARASWATI, 2004).

0 Yoga aponta caminhos de transformação e expansão da consciência em todos as dimensões do ser humano. As posturas do Yoga servem como veículos ao longo dessa jornada, e não como fins em si mesmas (JOSEPH \& LILIAN LE PAGE, 2015).

Para Taimni, o Yoga é a técnica real de autodisciplina, autodescoberta e autorealização, que tem como objetivo a completa e permanente libertação do homem das limitações nas quais está envolvido (TAIMNI, 2012).

0 Yoga libera o potencial criativo da vida, e faz isso estabelecendo uma estrutura para a auto-realizaçao, mostrando como podemos avançar na jornada. A luz que oYoga lança na vida é especial, é transformadora, não muda apenas nosso modo de ver as coisas, ela transforma a pessoa que vê. Traz conhecimento e o eleva a condição de sabedoria (IYENGAR et al.,2016).

Para o Ministério da saúde, entre os principais benefícios obtidos por meio da prática do yoga estão a redução do estresse, a regulação do sistema nervoso e respiratório, o equilíbrio do sono, o aumento da vitalidade psicofísica, o equilíbrio da produção hormonal, o fortalecimento do sistema imunológico,o aumento da capacidade de concentração e de criatividade e a promoção da reeducação mental com consequentemelhori adosquadrosdehumor,oquereverberanaqualidadedevi dados praticantes (BRASIL,2017).

Diante de tantas insatisfações e aflições que envolve o ser humano, o Yoga tem sido utilizada com a finalidade de minimizar ansiedade, stress e promover o bem estar 
no indivíduo. Tem sido comprovado através de pesquisas científicas resultados positivos e concretos. 0 objetivo desta pesquisa foi demonstrar através da prática do Yoga, efeitos positivos frente a ansiedade e a qualidade de vida.

\section{METODOLOGIA}

Tratou-se de uma pesquisa de estudo longitudinal, comparativo de abordagem quantitativa, realizado na populaçao composta por mulheres funcionárias de uma empresa de direito com queixa de ansiedade.

Foram incluídas no estudo funcionárias mulheres com queixa de ansiedade e foram excluídas mulheres funcionárias da empresa com problemas na coluna, hipertensão arterial, diabetes e quem estivesse em uso de psicotrópicos que podiam interferir no equilíbriofísico.

0 estudo foi realizado em uma Empresa de Advocacia em Sapucaia do Sul, no período de 22 de setembro de 2020 a 29 de outubro de 2020.

A divulgação aconteceu por meio de uma palestra na empresa (Figuras 1) que foi realizada no dia 16 de setembro de 2020, com o tema sobre Ansiedade, Yoga e qualidade de vida. Também foram dadas as informações sobre a pesquisa que seria realizada e questionários que teriam que responder.

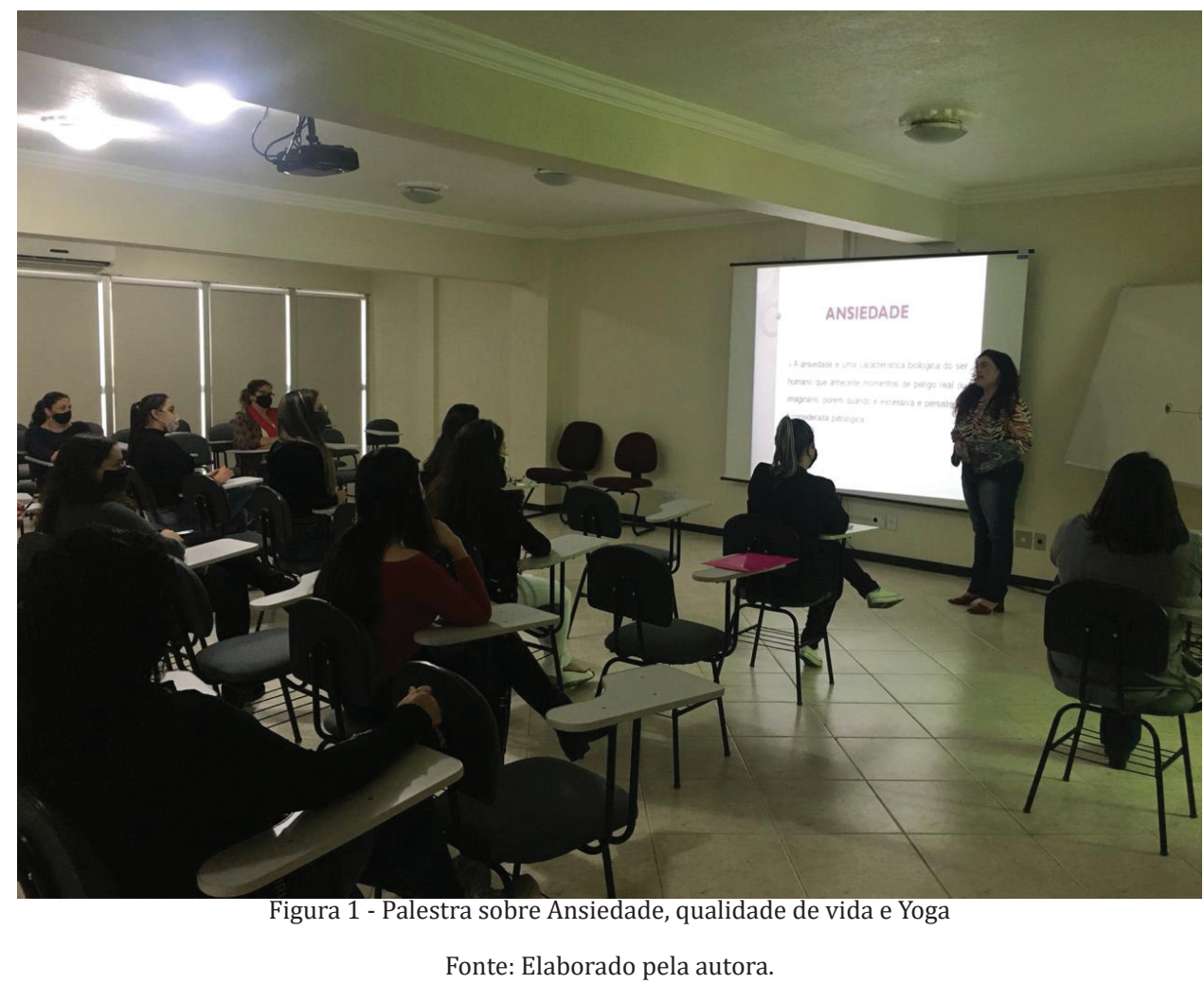

No dia da palestra foi entregue uma lista para que as funcionárias interessadas em participarem da pesquisa, para que deixassem o nome e contato para marcar a entrevista que foi realizada no dia seguinte, dia 17 de setembro durante todo o dia.

Para coleta de dados as participantes responderam dois questionários: 0 inventário de ansiedade de Beck, para verificar o nível de ansiedade; o questionário Whoqol-Bref, para verificar o grau de qualidade de vida, ambos foram aplicados antes e depois da intervenção, e para caracterizar a população, foi respondido um ques- tionário socioeconômico na entrevista, o tempo utilizado para responder os questionários foi uma média de 30 minutos. As entrevistas para responderem os questionários e as práticas de Yoga, foram realizadas em sala específica cedida pela empresa.

A intervenção ocorreu do dia 22 de setembro a 29 de outubro de 2020. As práticas de Yoga (Figuras 2, 3 e 4) tiveram a duração de um mês e meio, sendo realizadas 12 aulas com o tempo de 1(uma) hora de duração, com a frequência de duas vezes por semana, toda terça-feira e quinta-feira. 


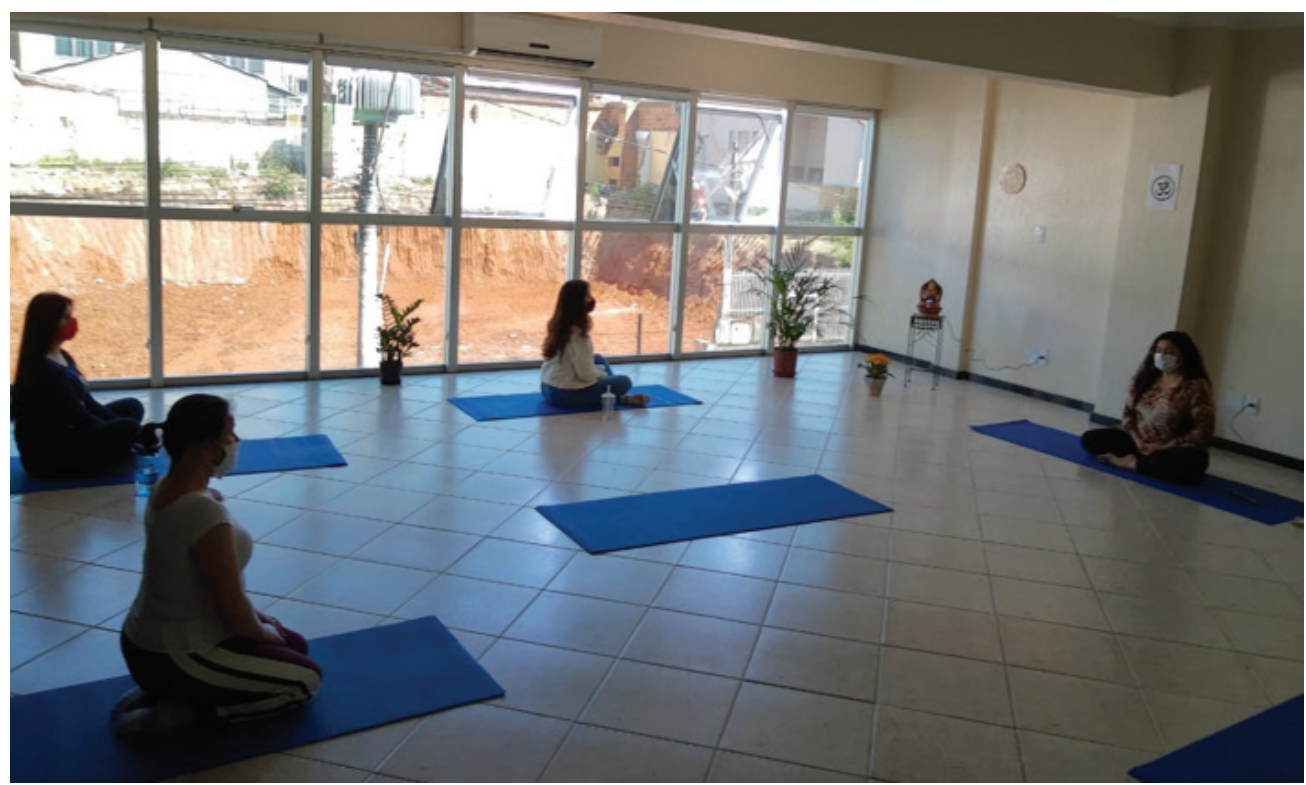

Figura 2 - Postura de Yoga ArdhaPadmasana

Fonte: Elaborado pela autora.

As aulas foram compostas por àsanas (posturas que trabalham corpo e mente), permanência, técnicas respiratórias, meditação e relaxamento. As voluntarias foram orientadas a buscar consciência corporal, realizar movimentos suaves e fluidos, sempre respeitando o limite do corpo, sem forçar e nem alongar demasiadamente. Buscar consciência respiratória e assim ter os efeitos benéficos da respiração completa no corpo e na mente e eliminar tensões desnecessárias.

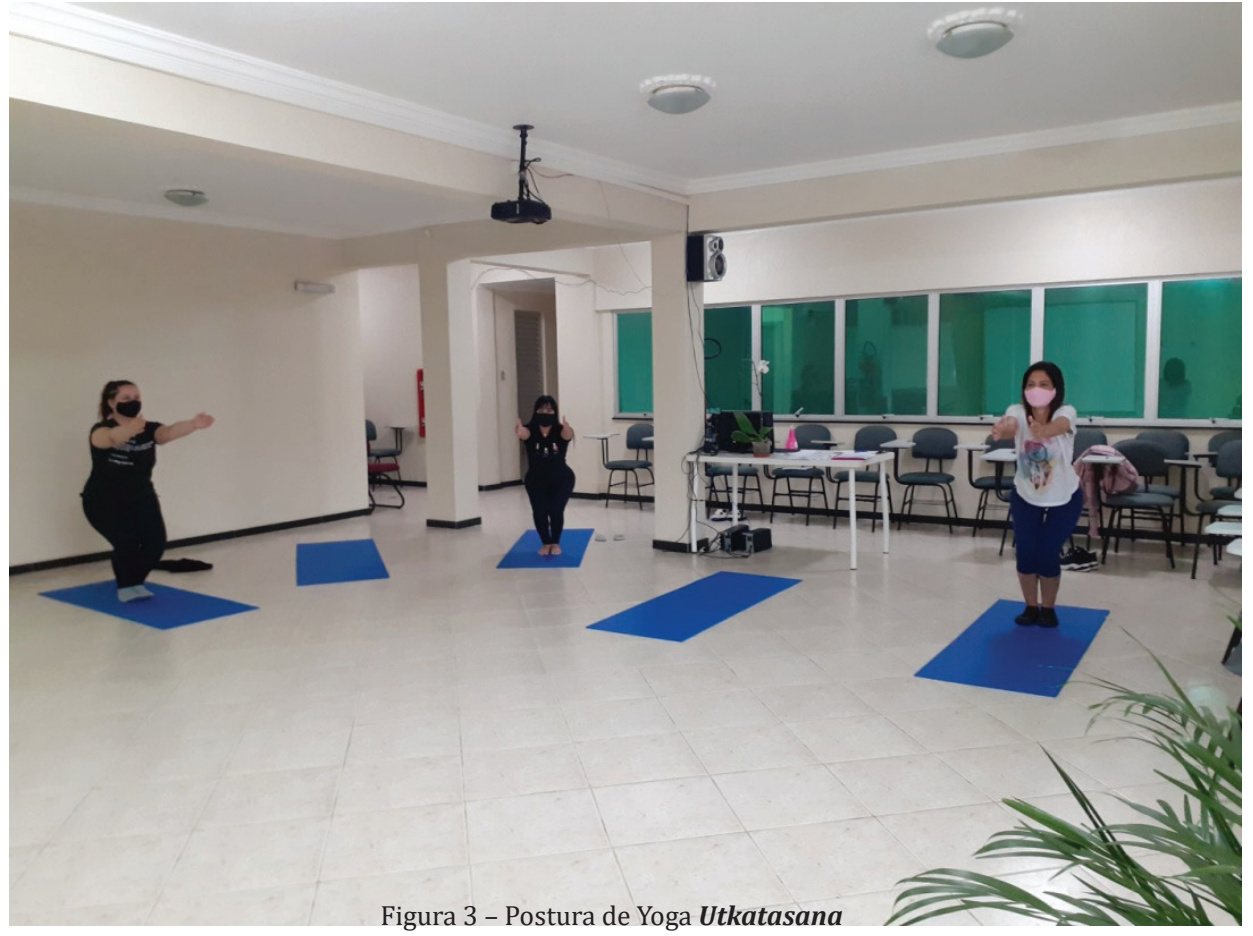

Fonte: Elaborado pela autora. 


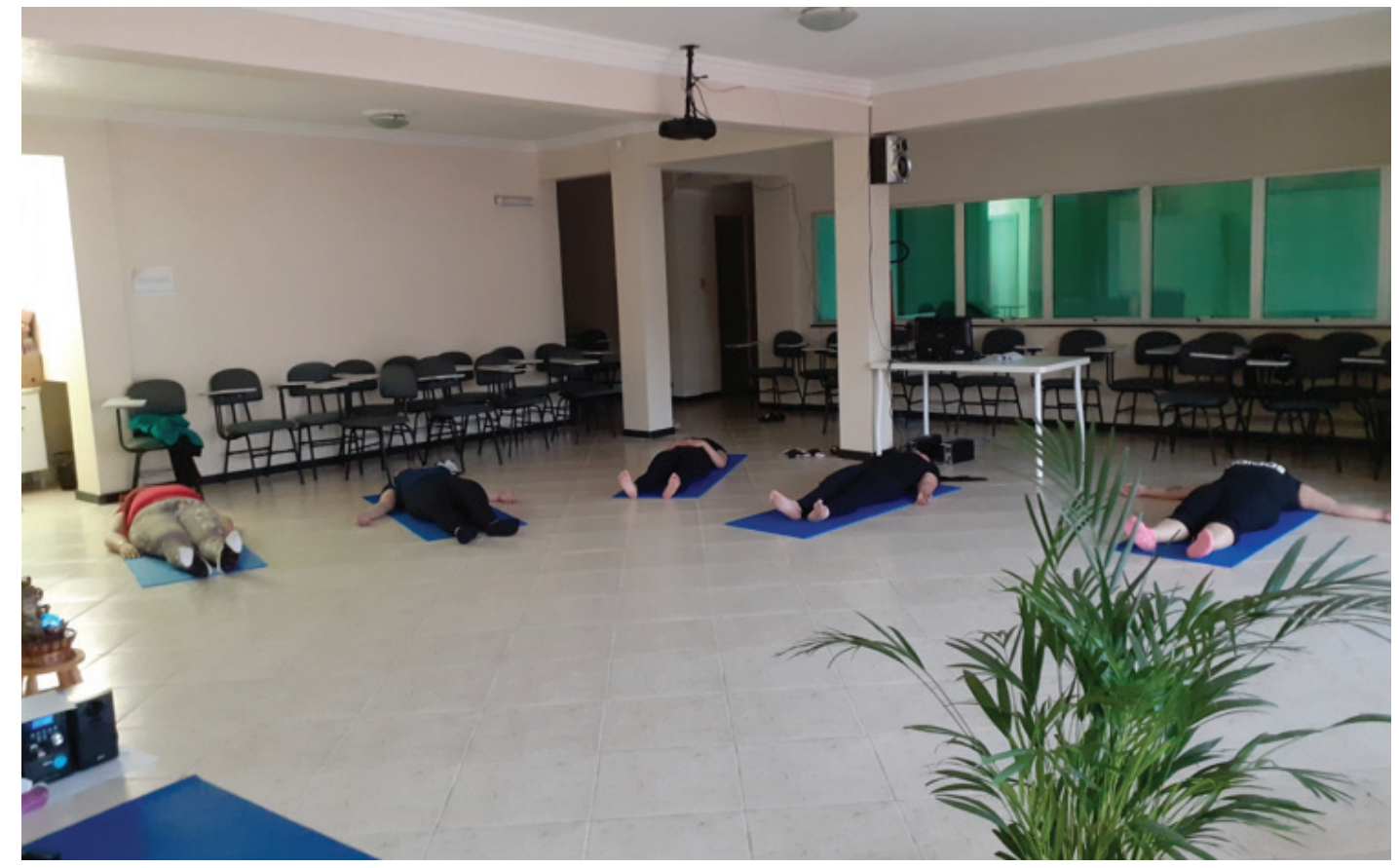

Figura 4 - Postura de Yoga para Relaxamento

Fonte: Elaborado pela autora.

Considerando o momento em que estamos vivendo

nesta pandemia, todas as medidas restritivas e preventivas necessárias para proteção dos participantes e da pesquisadora foram tomadas. Foi obrigatório o uso de máscaras, a sala onde foram realizadas todas as práticas era espaçosa e permitiu o distanciamento de dois metros um do outro, as participantes foram divididasem três turmas, portanto foi realizado 3 horários de turmas semanalmente. Foiobrigatório o uso do álcool gel nas mãos na entrada do ambiente, todos os tapetes foram utilizados individualmente e qualquer material utilizado em sala, foram higienizadoscom álcool 70\%. Foi distribuído no primeiro dia de aula para cada participante, um protocolocontendo orientações e cuidados na prevenção do coronavírus na sala de prática de Yoga.

\section{ANÁLISE ESTATÍSTICA}

As análises foram realizadas no programa estatístico SPSS 22.0 (SPSS Inc., EUA). A normalidade dos dados foi avaliada através do teste de Shapiro-Wilk, e os valores foram apresentados em média +- desvio padrão. Um teste $t$-Student para dados independentes foi realizado para avaliar os efeitos da intervenção. Um nível de significância de $\mathrm{p}<0,05$ foi adotado para todas as análises.

\section{RESULTADOS}

Esta pesquisa foi realizada com 12 participantes mulheres funcionárias de uma empresa de advocacia com queixa de ansiedade que participaram desde o início ao término da pesquisa, respondendo a dois questionários de avaliação antes e no final das práticas, um questionário socioeconômico e participando das aulas/ práticas semanais durante um mês e meio, no total de 12 aulas.

Nos dados socioeconômicos, descrevemos a caracterização da população e ressaltamos a idade das participantes, 66,7\% tinham idade de 20 a 30 anos e 33,3\% de 40 a 55 anos, os outros dados estão também expostos na tabela a baixo: 
Tabela 1 - Caracterização Sócio Demográfica da Amostra

\begin{tabular}{|c|c|}
\hline \multicolumn{2}{|l|}{ Idade } \\
\hline - $\quad 20$ a 30 anos & $66,7 \%$ \\
\hline - $\quad 40$ a 55 anos & $33,3 \%$ \\
\hline Gênero (feminino) & $100 \%$ \\
\hline Residência própria & $100 \%$ \\
\hline \multicolumn{2}{|l|}{ Mora junto } \\
\hline - 1 & $16,7 \%$ \\
\hline - 2 & $25 \%$ \\
\hline - 3 & $33,3 \%$ \\
\hline - 4 & $8,3 \%$ \\
\hline - 5 & $16,7 \%$ \\
\hline Escolaridade & \\
\hline - Ensino Médio Incompleto & $8,3 \%$ \\
\hline - Ensino Médio Completo & $16,7 \%$ \\
\hline - Ensino Superior Incompleto & $66,7 \%$ \\
\hline - Ensino Superior Completo & $8,3 \%$ \\
\hline \multicolumn{2}{|l|}{ Profissão } \\
\hline - Auxiliar de escritório & $41,7 \%$ \\
\hline - Secretaria & $8,3 \%$ \\
\hline - $\quad$ Auxiliar de recepção & $8,3 \%$ \\
\hline - $\quad$ Advogada & $8,3 \%$ \\
\hline - $\quad$ Estagiária & $16,7 \%$ \\
\hline - Auxiliar jurídico & $8,3 \%$ \\
\hline $\begin{array}{l}\text { - Serviços gerais } \\
\text { Renda mensal }\end{array}$ & $8,3 \%$ \\
\hline - $\quad 1$ - 2 salários mínimos & $91,7 \%$ \\
\hline - $\quad 3$ - 4 salários mínimos & $8,3 \%$ \\
\hline Feliz com atividade laboral & $91,7 \%$ \\
\hline
\end{tabular}

Dados apresentados em frequência relativa.

Fonte: Elaborado pela autora.

A análise dos resultados do gráfico do Inventário de ansiedade de Beck (Figura 5), apresentou um quadro significativo na diminuição da ansiedade em todas as participantes. No gráfico é possível verificar $\mathrm{p}<0,01$ demonstrando uma pontuação bem alta no padrão de ansiedade antes da prática de Yoga, e a baixa dessa pontuação após as práticas de Yoga, comprovando a diminuição do padrão de ansiedade.

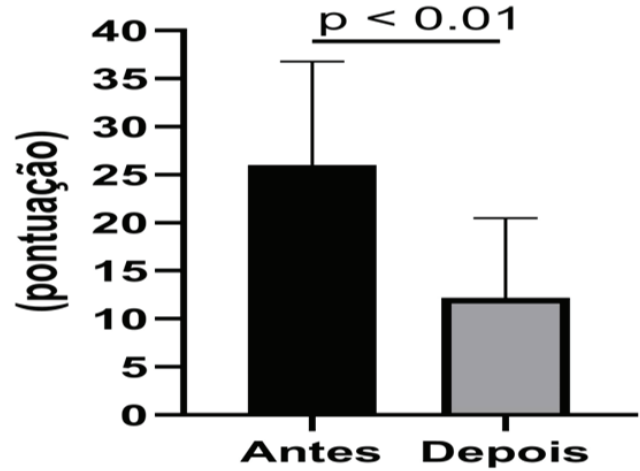

Figura 5 - Dados obtidos através do Inventário de Ansiedade de Beck

Fonte: Elaborado pela autora.

Na análise da qualidade de vida, através do questionário Whoqol-bref, foram encontrados os seguintes resultados (Tabela 2): obtivemos valores maiores no domínio físico $(\mathrm{p}<0,045)$, no meio ambiente $(\mathrm{p}<0,041)$ e na auto avaliação $(\mathrm{p}<0,05)$ QV após as práticas do Yoga.

Tabela 2 - Resultado obtidos através do Questionário Whoqol-bref

\begin{tabular}{lccc}
\hline & Antes & Depois & $\mathrm{p}$ \\
\hline $\begin{array}{l}\text { Domínio } \\
\text { Físico }\end{array}$ & $12,43 \pm 1,31$ & $14,19 \pm 1,40$ & $\mathbf{0 , 0 4 5}$ \\
$\begin{array}{l}\text { Domínio } \\
\text { Psicológico }\end{array}$ & $13,33 \pm 1,82$ & $13,67 \pm 2,10$ & 0,34 \\
$\begin{array}{l}\text { Relações } \\
\text { Sociais }\end{array}$ & $14,89 \pm 3,05$ & $15,67 \pm 2,61$ & 0,08 \\
$\begin{array}{l}\text { Meio } \\
\begin{array}{l}\text { Ambiente } \\
\text { Auto- } \\
\text { avaliação QV }\end{array}\end{array}$ & $12,04 \pm 2,64$ & $14,92 \pm 1,66$ & $\mathbf{0 , 0 4 1}$ \\
Geral & $13,14 \pm 1,45$ & $14,50 \pm 1,47$ & 0,13 \\
\hline
\end{tabular}

Dados apresentados em média \pm desvio padrão. Comparação antes - depois da intervenção através de um Teste $t$-Student para dados independentes $(\mathrm{p}<0,05)$. 


\section{DISCUSSÃo}

O presente estudo buscou investigar as contribuições do Yoga frente à ansiedade e a qualidade de vida. Seus resultados foram positivos e sugerem que a prática de Yoga contribui para a diminuição da ansiedade e a melhora na qualidade de vida. De acordo com o inventário de ansiedade de Beck, ouve uma redução significativa do grau de ansiedade. No início da pesquisa, conforme as respostas do inventário de ansiedade de Beck, muitas participantes tinham graus elevados de ansiedade e após a intervenção com Yoga o grau de praticamente todas as participantes foram reduzidos.

Vorkapic e Rangé relatam 8 investigações de ensaios controlados que observaram os efeitos do Yoga na ansiedade, e quase todos relataram que intervenção do Yoga diminui consideravelmente os níveis de ansiedade e estresse quando comparado ao grupo controle (VORKAPIC; RANGÉ, 2011). Correa e colaboradores(2020), conclui em seu trabalho, que os dados observacionais sugerem que praticantes mais experientes e que realizam um maior número de sessões semanal durante a pandemia de COVID-19 reportam menor impacto psicológico. Recomendações para a continuação da prática diária do Yoga pode contribuir para o controle da saúde física e mental durante esse momento de pandemia (CORREA et al., 2020).

PARTHASARATHY et al, na pesquisa realizada na índia com mulheres com problemas de ansiedade comprovada, concluíram que a prática de Yoga diminuiua pontuação da ansiedade sem ter quaisquer efeito nocivo (PARTHASARATHY et al,2014). BERNARDI et al, avaliaram o efeito do Yoga no nível de estresse e ansiedade em mulheres mastectomizadas, os dados encontrados são estatisticamente significantes e demonstraram que a intervenção com Yoga diminuiu o estresse e a ansiedade no grupo experimental (BERNARDI et al, 2013)

PONTE e colaboradores, em seu estudo intitulado Yoga nos cuidados primários de saúde, um estudo quase experimental para avaliar os efeitos na qualidade de vida e sofrimento psicológico analisaram que o Yoga melhora significativamente a qualidade de vida e reduz o sofrimento psicológico. 0 Yoga na atenção básica é viável, seguro e tem adesão satisfatória, além de repercutir positivamente na qualidade de vida e na redução do sofrimento psicológico dos participantes (PONTE et al., 2019). GALLAGHER et al, em um estudo de ensaio clínico randomizado sobre efeitos do Yoga na ansiedade e depressão em mulheres grávidas em repouso no leito hospitalar, solicitado por médico, concluíram que os resultados demonstraram que o Yoga é uma intervenção eficaz para diminuição da ansiedade e depressão em mulheres pré-parto de alto riscos em repouso no leito hospitalar (GALLAGHER et al.,2019)

ALMEIDA e colaboradores, utilizaram em sua pesquisa influência do hatha-yoga na qualidade de vida de mulheres o Whoqol-bref, a conclusão foi que a prática sistemática do Hatha-Yoga influenciou de forma positiva a qualidade de vida, pois as mulheres com mais tempo de prática apresentaram qualidade de vida melhor do que aquelas com menos tempo de prática (ALMEIDA et al., 2017).

Em nossa pesquisa, noWhoqol-bref ouve melhora em três domínios, ofísico que está relacionado a energia e fadiga, sono e repouso (houve o relato em sala de aula de duas alunas que tinham o histórico de não conseguirem dormir, e desde o início das aulas de yoga estavam conseguindo dormir), capacidade de trabalho, dentre outros. Omeio ambiente, está ligado ao ambiente no lar, ambiente físico, oportunidades de participar de recreação e lazer, cuidados de saúde e sociais, dentre outros. A auto avaliação da qualidade de vida, que é a percepção sobre a qualidade de vida global e percepção geral de saúde. A prática de Yoga contribui para a melhora no grau da qualidade de vida, sendo demonstrado pelas respostas do whoqo-bref aplicado antes das práticas do Yoga, e das respostas do whoqol-bref aplicado após as práticas do Yoga.

Neste estudo foi possível comprovar através dos resultados a eficácia do Yoga frente a ansiedade e a qualidade de vida. Assim, o Yoga pode ser considerado uma ferramenta que auxilia na diminuição da ansiedade e na melhora da qualidade de vida. Esperamos contribuir com o desenvolvimento de estratégias na diminuição da ansiedade e melhora na qualidade de vida das pessoas.

\section{CONCLUSÃO}

Esta pesquisa nos permite concluir que existe uma diferença significativa entre os níveis de ansiedade e a qualidade de vida antes e depois da intervenção com as práticas de Yoga. Por meio dos resultados obtidos neste estudo, foi possível demonstrar que a prática de Yoga diminuiu consideravelmente o grau de ansiedade e contribuiu para uma melhora na qualidade de vida dos praticantes. Considerando nossos resultados e outros estudos científicos, a prática do Yoga deve ser incentivada como uma prática que traz grandes benefícios a saúde e a vida das pessoas. 


\section{REFERÊNCIAS}

ALMEIDA, Marcos Bezerra et al. INFLUÊNCIA DO HATHA-YOGA NA QUALIDADE DE VIDA DE MULHERES. In: Congresso Internacional de Atividade Física, Nutrição e Saúde. 2017.

BARROS, Nelson Filice de et al. Yoga e promoção da saúde. Ciência \& Saúde Coletiva, v. 19, p. 1305-1314, 2014.

BERNARDI, Marina Lima Daleprane et al. Efeitos da intervenção Hatha-Yoga nos níveis de estresse e ansiedade em mulheres mastectomizadas. Ciência \& Saúde Coletiva, v. 18, p. 3621 $3632,2013$.

BORELLA, Ana et al. 0 livro de ouro do yoga. Rio de Janeiro: Ediouro, 2007

BRASIL. Ministério da Saúde. Práticas Integrativas e Complementares (PICS): quais são e para que servem. Brasília, DF: Ministério da Saúde, 2017. Disponível em: https://saude.gov. $\mathrm{br} /$ saude-de-a-z/praticas-integrativas-e-complementares. Acesso em: 05 mar. 2020

CORREA, Cinthia Andriota et al. Níveis de estresse, ansiedade, depressão e fatores associados durante a pandemia de COVID-19 em praticantes de Yoga. Revista Brasileira de Atividade Física \& Saúde, v. 25, p. 1-7, 2020.

DANUCALOV, Marcello Árias Dias. Efeitos psicofisiológicos de um programa de yoga e meditação para redução de estresse em cuidadores de pacientes com Alzheimer. 2012. 165 f. Tese (Doutorado) - Escola Paulista de Medicina, Universidade Federal de São Paulo. São Paulo, 2012.

GALLAGHER A, Kring D, Whitley T. Effects of yoga on anxiety and depression for high risk mothers on hospital bedrest. Complement TherClinPract. 2020 Feb;38:101079. doi: 10.1016/j.ctcp.2019.101079. Epub 2019 Dec 14. PMID: 32056815

GRACIOLI, Júlia. Brasil vive surtos de depressão e ansiedade. Jornal da USP, São Paulo, paginação irregular, ago. 2018. Disponível em: https://jornal.usp.br/atualidades/brasil-vive-surto-de-depressao-e-ansiedade/. Acesso em: 05 mar. 2020.
GUIMARÃES, Claudia Maria. Efeito da acupuntura nos sintomas de ansiedade e depressão e nos parâmetros fisiológicos de voluntários adultos. 2007. Tese (Doutorado em Ciências) - Universidade Federal de São Paulo, Escola Paulista de Medicina, São Paulo, 2007.

IYENGAR, B. K. S. et al. Luz sobre o yoga: o guia clássico de yoga escrito pelo embaixador do yoga no ocidente. São Paulo: Pensamento, 2016

JOSEPH, W.; PAGE, L. Guppy - Guia prático de posturas de Yoga. Yoga Integrativa, Montanha Encantada, SC, 2015

LEAHY, Robert L., Livre de ansiedade, Porto Alegre: Artmed, 2010.

PARTHASARATHY, S. et al. Effect of integrated yoga module on selected psychological variables among women with anxiety problem. West Indian Medical Journal, v. 63, n. 1, p. 78-80, 2014.

PONTE, Sara B. et al. Yoga in primaryhealthcare: A quasi-experimental studyto Access theeffectsonqualityoflifeandpsychologicaldistress. Complementarytherapies in clinicalpractice, v. 34, p. 1-7, 2019.

SARASWATI,SwamiSatyananda.AsanaPranayamaMudra Bandha, $3^{\circ}$ ediçao revisada. New Delhi Yoga Publications Trust,Munger, Bihar, India, 2004.

SILVA, Ana Beatriz B. Mentes ansiosas: medo e ansiedade nossos de cada dia. 2. ed. São Paulo: Principium, 2017.

STILES, Tara. A cura pelo yoga: rotina simples para superar mais de 50 problemas de saúde e viver sem dor. São Paulo: Pensamento, 2014.

TAIMNI, L. K. Preparação para o yoga. 3. ed. Brasília, DF: Teosófica, 2012.

VORKAPIC, Camila Ferreira; RANGÉ, Bernard. Os benefícios do yoga nos transtornos de ansiedade. Revista Brasileira de Terapias Cognitivas, v. 7, n. 1, p. 50-54, 2011. 